\title{
Dirac charge dynamics in graphene by infrared spectroscopy
}

\author{
Z. Q. LI ${ }^{1 *}$, E. A. HENRIIKSEN², Z. JIANG ${ }^{2,3}$, Z. HAO4 ${ }^{4}$, M. C. MARTIN4, P. KIM², H. L. STORMER ${ }^{2,5,6}$ \\ AND D. N. BASOV ${ }^{1}$
}

\author{
${ }^{1}$ Department of Physics, University of California, San Diego, La Jolla, California 92093, USA \\ ${ }^{2}$ Department of Physics, Columbia University, New York 10027, USA \\ ${ }^{3}$ National High Magnetic Field Laboratory, Tallahassee, Florida 32310, USA \\ ${ }^{4}$ Advanced Light Source Division, Lawrence Berkeley National Laboratory, Berkeley, California 94720, USA \\ ${ }^{5}$ Department of Applied Physics and Applied Mathematics, Columbia University, New York 10027, USA \\ ${ }^{6}$ Bell Labs, Alcatel-Lucent, Murray Hill, New Jersey 07974, USA \\ *e-mail: zhiqiang@physics.ucsd.edu
}

A remarkable manifestation of the quantum character of electrons in matter is offered by graphene, a single atomic layer of graphite. Unlike conventional solids where electrons are described with the Schrödinger equation, electronic excitations in graphene are governed by the Dirac hamiltonian ${ }^{1}$. Some of the intriguing electronic properties of graphene, such as massless Dirac quasiparticles with linear energy-momentum dispersion, have been confirmed by recent observations ${ }^{2-5}$. Here, we report an infrared spectromicroscopy study of charge dynamics in graphene integrated in gated devices. Our measurements verify the expected characteristics of graphene and, owing to the previously unattainable accuracy of infrared experiments, also uncover significant departures of the quasiparticle dynamics from predictions made for Dirac fermions in idealized, freestanding graphene. Several observations reported here indicate the relevance of many-body interactions to the electromagnetic response of graphene.

We investigated the reflectance $R(\omega)$ and transmission $T(\omega)$ of graphene samples on a $\mathrm{SiO}_{2} / \mathrm{Si}$ substrate (inset of Fig. 1a) as a function of gate voltage $V_{\mathrm{g}}$ at $45 \mathrm{~K}$ (see the Methods section). We start with data taken at the charge-neutrality point $V_{\mathrm{CN}}$ : the gate voltage corresponding to the minimum d.c. conductivity and zero total charge density (inset of Fig. 1c). Figure 1a shows $R(\omega)$ of a graphene gated structure (graphene $/ \mathrm{SiO}_{2} / \mathrm{Si}$ ) at $V_{\mathrm{CN}}=3 \mathrm{~V}$ normalized by reflectance of the substrate $R_{\text {sub }}(\omega) . R_{\text {sub }}(\omega)$ is dominated by a minimum around $5,500 \mathrm{~cm}^{-1}$ due to interference effects in $\mathrm{SiO}_{2}$. A remarkable observation is that a monolayer of undoped graphene markedly modifies the interference minimum of the substrate leading to a suppression of $R_{\text {sub }}(\omega)$ by as much as $15 \%$. This observation is significant because it enables us to evaluate the conductivity of graphene near the interference structure, as will be discussed below.

Both reflectance and transmission spectra of graphene structures can be modified by a gate voltage. Figure $1 \mathrm{~b}, \mathrm{c}$ shows these modifications at various gate voltages normalized by data at $V_{\mathrm{CN}}: R(V) / R\left(V_{\mathrm{CN}}\right)$ and $T(V) / T\left(V_{\mathrm{CN}}\right)$, where $V=V_{\mathrm{g}}-V_{\mathrm{CN}}$. These data correspond to the Fermi energy $E_{\mathrm{F}}$ on the electron side and similar behaviour was observed with $E_{\mathrm{F}}$ on the hole side (not shown). At low voltages $(<17 \mathrm{~V})$, we found a dip in $R(V) / R\left(V_{\mathrm{CN}}\right)$ spectra. With increasing bias, this feature evolves into a peak-dip structure and systematically shifts to higher frequency. The $T(V) / T\left(V_{\mathrm{CN}}\right)$ spectra reveal a peak at all voltages, which systematically hardens with increasing bias. A voltage-induced increase in transmission $\left(T(V) / T\left(V_{\mathrm{CN}}\right)>1\right)$ signals a decrease of the absorption with bias. Most interestingly, we observed that the frequencies of the main features in $R(V) / R\left(V_{\mathrm{CN}}\right)$ and $T(V) / T\left(V_{\mathrm{CN}}\right)$ all evolve approximately as $\sqrt{V}$.

To explore the quasiparticle dynamics under applied voltages, it is imperative to first discuss the two-dimensional (2D) optical conductivity of charge-neutral graphene, $\sigma_{1}\left(\omega, V_{\mathrm{CN}}\right)+i \sigma_{2}\left(\omega, V_{\mathrm{CN}}\right)$, extracted from a multilayer analysis of the devices (see the Methods section). Theoretical analysis ${ }^{6-8}$ predicts a constant 'universal' $2 \mathrm{D}$ conductivity $\sigma_{1}\left(\omega, V_{\mathrm{CN}}\right)=\pi e^{2} / 2 h$ for ideal undoped graphene. Our $R(\omega) / R_{\text {sub }}(\omega)$ data are consistent with this prediction. Figure 1a shows a comparison between the experimental $R(\omega) / R_{\text {sub }}(\omega)$ spectrum and model spectra generated assuming constant $\sigma_{1}\left(\omega, V_{\mathrm{CN}}\right)$ values. The constant universal conductivity offers a good agreement (within $\pm 15 \%$ ) with the experimental spectra in the range $4,000-6,500 \mathrm{~cm}^{-1}$. Outside this spectral region, our infrared measurements do not allow us to unambiguously determine the absolute value of $\sigma_{1}\left(\omega, V_{\mathrm{CN}}\right)$; therefore, the uncertainty of $\sigma_{1}\left(\omega, V_{\mathrm{CN}}\right)$ increases as shown by the shaded region weighted around the $\pi e^{2} / 2 h$ value in Fig. $2 \mathrm{a}$. However, recent infrared studies of graphene revealed a constant conductivity $\sigma_{1}\left(\omega, V_{\mathrm{CN}}\right)=\pi e^{2} / 2 h$ between 2,400 and $24,000 \mathrm{~cm}^{-1}$ (ref. 9 and Mak, K.F. \& Heinz, T. 2008 APS March Meeting, Abstract: L29.00006, unpublished). The universal conductivity is only weakly modified in bulk highly ordered pyrolytic graphite ${ }^{10}$ and extends down to $800 \mathrm{~cm}^{-1}$. Thus, in the following discussion, we will assume $\sigma_{1}\left(\omega, V_{\mathrm{CN}}\right)=\pi e^{2} / 2 h$ throughout the entire range of our data.

Electrostatic doping of graphene introduces two fundamental changes in the optical conductivity $\sigma_{1}(\omega, V)+i \sigma_{2}(\omega, V)$ : a strong Drude component formed in the far-infrared with $\sigma_{1}(\omega \rightarrow 0)=4-100 \pi e^{2} / 2 h$ accompanied by a shift in the onset of interband transitions at $2 E_{\mathrm{F}}$, as schematically shown in the inset of Fig. 2b. To investigate these effects, we obtained 


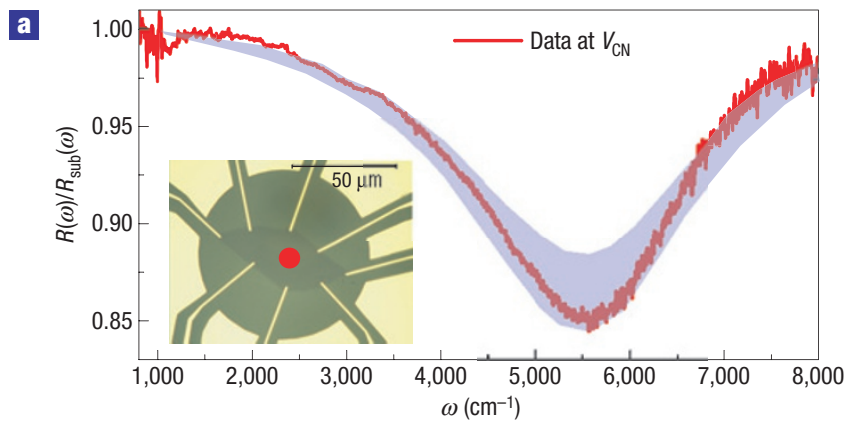

b

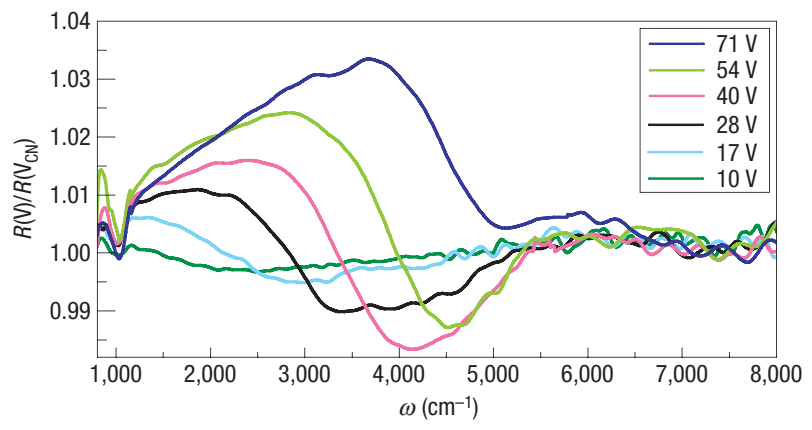

c

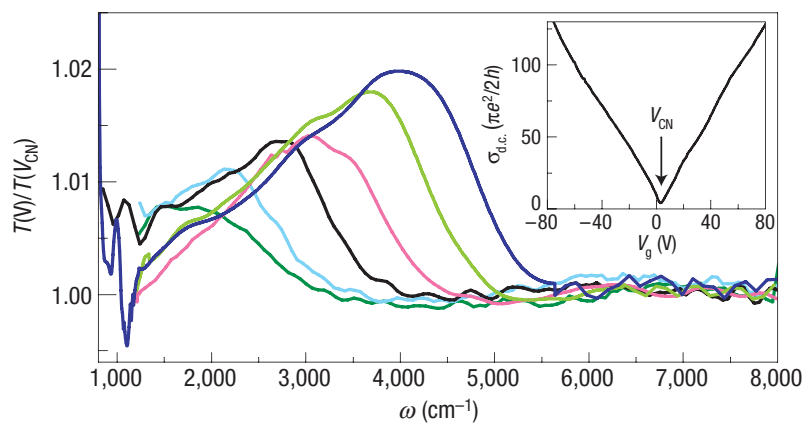

Figure 1 The reflectance $R(\omega)$ and transmission $T(\omega)$ of a graphene device under applied gate voltages. a, The reflectance of the graphene device (graphene/SiO $\left.{ }_{2} / \mathrm{Si}\right) R(\omega)$ normalized by that of the $\mathrm{SiO}_{2} / \mathrm{Si}$ substrate $R_{\text {sub }}(\omega)$ at the charge-neutrality voltage $V_{\text {CN. }}$. A set of $R(\omega) / R_{\text {sub }}(\omega)$ spectra generated from the multilayer model using a constant $\sigma_{1}\left(\omega, V_{\mathrm{CN}}\right)$ in the range of $(1 \pm 0.15) \pi e^{2} / 2 h$ are shown as the shaded area. The upper and lower boundary of the shaded area are defined by $\sigma_{1}\left(\omega, V_{\mathrm{CN}}\right)$ with values of $0.85^{*} \pi e^{2} / 2 h$ and $1.15^{*} \pi e^{2} / 2 h$, respectively. Inset: A photograph of a graphene device together with a schematic diagram of the focused synchrotron beam (red dot). b,c, $R(V) / R\left(V_{\mathrm{CN}}\right)(\mathbf{b})$ and $T(V) / T\left(V_{C N}\right)$ (c) spectra of the graphene device at several voltages corresponding to $E_{\mathrm{F}}$ on the electron side, where $V=V_{\mathrm{g}}-V_{\mathrm{CN}}$. The inset of $\mathbf{c}$ shows the smoothed d.c. conductivity data of the sample as a function of gate voltage $V_{\mathrm{g}}$.

$\sigma_{1}(\omega, V)+i \sigma_{2}(\omega, V)$ (Fig. 2b,c) from voltage-dependent reflectance and transmission spectra (see the Methods section). The key features in the conductivity spectra are independent of uncertainties in $\sigma_{1}\left(\omega, V_{\mathrm{CN}}\right)$ discussed above. Regardless of the choice of $\sigma_{1}\left(\omega, V_{\mathrm{CN}}\right)$, under applied biases, we observe a suppression of the conductivity compared with $\sigma_{1}\left(\omega, V_{\mathrm{CN}}\right)$ and a well-defined threshold structure above which the conductivity recovers the universal value $\pi e^{2} / 2 h$. The energy of the threshold structure systematically increases with voltage, a natural expectation for a transition occurring at $2 E_{\mathrm{F}}$. With a scattering rate $1 / \tau=30 \mathrm{~cm}^{-1}$ at $71 \mathrm{~V}$ independently obtained from transport data, the Drude mode is narrow and confined

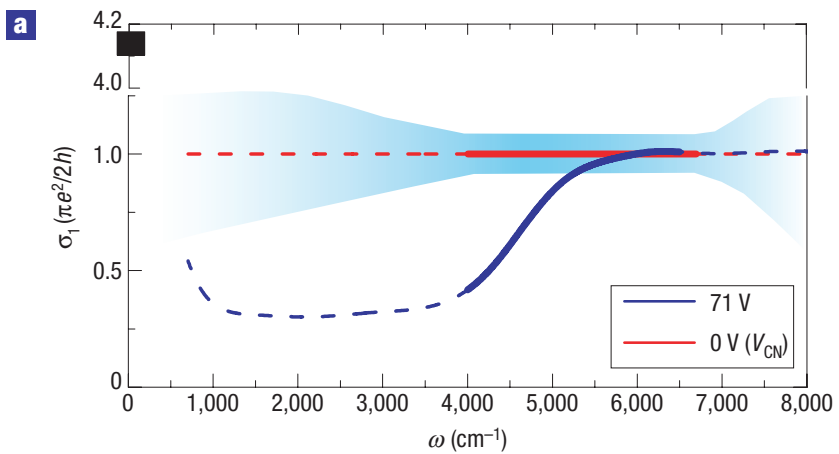

b

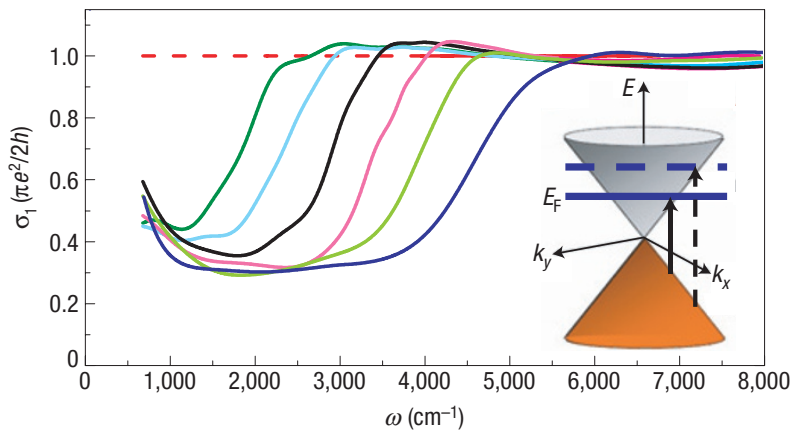

G

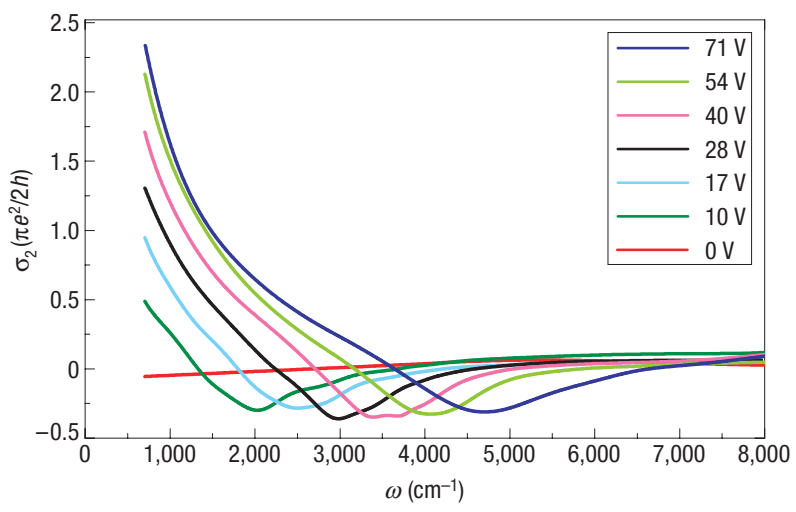

Figure 2 The optical conductivity of graphene at different voltages. a, The real part of the 2D optical conductivity $\sigma_{1}(\omega)$ at $V_{\mathrm{CN}}$ and $71 \mathrm{~V}$. The solid red line shows the region where our data support the universal result. The uncertainty of $\sigma_{1}\left(\omega, V_{\mathrm{CN}}\right)$ is shown by the shaded area with the theoretical $\sigma_{1}(\omega)=\pi e^{2} / 2 h$ plotted as the red dashed line. The blue dashed line is $\sigma_{1}(\omega)$ at $71 \mathrm{~V}$ evaluated for the theoretical spectra: $\sigma_{1}\left(\omega, V_{\mathrm{CN}}\right)=\pi e^{2} / 2 h$ (red dashed line). The key spectral features of $\sigma_{1}(\omega, V)$ are independent of uncertainties in $\sigma_{1}\left(\omega, V_{\text {CN }}\right)$ indicated by the shaded area, as discussed in the text. Black square on the left axis: d.c. conductivity at $V_{\mathrm{CN}}$. $\mathbf{b}, \mathbf{c}, \sigma_{1}(\omega)(\mathbf{b})$ and $\sigma_{2}(\omega)(\mathbf{c})$ of graphene at several voltages with respect to $V_{\mathrm{CN}}$ corresponding to $E_{\mathrm{F}}$ on the electron side based on $\sigma_{1}\left(\omega, V_{\mathrm{CN}}\right)=\pi e^{2} / 2 h$. The absolute values of the $\sigma_{2}(\omega)$ spectra in $\mathbf{c}$ have uncertainties due to the uncertainties of $\sigma_{1}\left(\omega, V_{\mathrm{CN}}\right)$ as discussed in the text. The inset of $\mathbf{b}$ shows the band structure of graphene near the Dirac point and the interband transition at $2 E_{\mathrm{F}}$.

below the low- $\omega$ cutoff of our measurements. We stress that the two voltage-induced transformations of the conductivity, the intraband mode and the onset of interband absorption at $2 E_{\mathrm{F}}$, are interdependent as suggested by our data. Indeed, assuming the intraband component can be described with a simple Drude formula $\sigma_{1}(\omega)=\sigma_{\text {d.c. }} /\left(1+\omega^{2} \tau^{2}\right)$ using $\sigma_{\text {d.c. }}$ and $1 / \tau$ obtained from transport measurements, we find that the spectral weight 


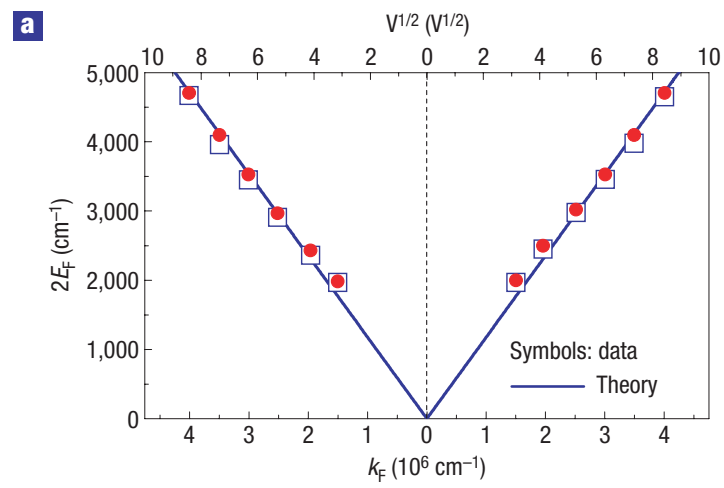

b

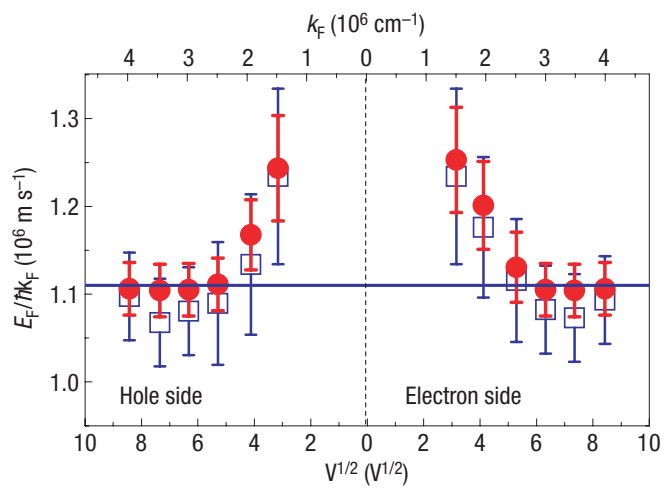

Figure 3 The Fermi energy $E_{\mathrm{F}}$ and the ratio of $E_{\mathrm{F}}$ to the Fermi wave vector $\boldsymbol{E}_{\mathrm{F}} / \boldsymbol{h} \boldsymbol{k}_{\mathrm{F}}$. The magnitude of $E_{\mathrm{F}} / \hbar k_{\mathrm{F}}$ is closely related to the Fermi velocity $v_{\mathrm{F}}$ as discussed in the text . a, The magnitude of $2 E_{\mathrm{F}}$ plotted as a function of $V^{1 / 2}$ and $k_{\mathrm{F}}$ for the electron and hole sides with respect to the charge-neutrality voltage. Red solid symbols: $2 E_{\mathrm{F}}$ extracted from the minimum in the $\sigma_{2}(\omega, V)$ spectra. Blue open symbols: $2 E_{\mathrm{F}}$ extracted from the centre of the $2 E_{\mathrm{F}}$ threshold in $\sigma_{1}(\omega, V)$. The uncertainties of the $2 E_{\mathrm{F}}$ values discussed in the Methods section do not exceed the size of the symbols. Solid lines are theoretical $2 E_{\mathrm{F}}$ values using $V_{\mathrm{F}}=1.11 \times 10^{6} \mathrm{~m} \mathrm{~S}^{-1} \cdot \mathbf{b}$, Symbols: $E_{\mathrm{F}} / \hbar k_{\mathrm{F}}$ as a function of $V^{1 / 2}$ and $k_{\mathrm{F}}$. The blue line corresponds to a $v_{\mathrm{F}}$ value of $1.11 \times 10^{6} \mathrm{~m} \mathrm{~S}^{-1}$. The error bars reflect uncertainties in the determination of the Fermi energy $2 E_{\mathrm{F}}$ from the conductivity data $\delta\left(E_{\mathrm{F}}\right)$ discussed in the Methods section and are calculated as $\delta\left(E_{\mathrm{F}} / \hbar k_{\mathrm{F}}\right)=\delta\left(E_{\mathrm{F}}\right) /\left(\hbar \sqrt{\pi C_{\mathrm{g}} V / e}\right)$.

removed from $\omega<2 E_{\mathrm{F}}$ is recovered under the Drude structure, such that the total oscillator strength given by $\int_{0}^{\Omega_{c}} \sigma_{1}(\omega) \mathrm{d} \omega$ is conserved at any bias with a cutoff frequency $\Omega_{\mathrm{c}}=8,000 \mathrm{~cm}^{-1}$.

Next, we extracted Fermi energy values from the $2 E_{\mathrm{F}}$ threshold using two different methods (see the Methods section). We found that the $2 E_{\mathrm{F}}$ values (Fig. 3a) are symmetric for biases delivering either holes or electrons to graphene. Moreover, $2 E_{\mathrm{F}}$ increases with voltage approximately as $\sqrt{V}$ (deviations from the square-root law at small biases will be discussed below). Note that $E_{\mathrm{F}}$ of Dirac fermions scales with the $2 \mathrm{D}$ carrier density $N$ as $E_{\mathrm{F}}=\hbar v_{\mathrm{F}} \sqrt{\pi N}$ (refs 2,3), where $v_{\mathrm{F}}$ is the Fermi velocity. In our devices, $N=C_{\mathrm{g}} V / e$, where $C_{\mathrm{g}}=115 \mathrm{aF} \mu \mathrm{m}^{-2}$ is the gate capacitance per unit area. Therefore, the observed $\sqrt{V}$ dependence of $2 E_{\mathrm{F}}$ substantiates that graphene samples integrated in gated devices are governed by Dirac quasiparticles.

Interestingly, the $2 E_{\mathrm{F}}$ threshold in $\sigma_{1}(\omega, V)$ shows a width of about $1,400 \mathrm{~cm}^{-1}$ that is independent of gate voltage and therefore of carrier density $N$, irrespective of a sevenfold enhancement of $N$ between 10 and $71 \mathrm{~V}$. This effect is much stronger than the theoretical estimate for thermal smearing of the $2 E_{\mathrm{F}}$ feature at $45 \mathrm{~K}$ (refs 7,8), which is about $500 \mathrm{~cm}^{-1}$. A recent theoretical study ${ }^{11}$ showed that disorder effects and electron-phonon coupling are needed to account for the width of the $2 E_{\mathrm{F}}$ threshold in our data. Apart from that, a spatial variation of local $E_{\mathrm{F}}$ values observed in graphene on $\mathrm{SiO}_{2} / \mathrm{Si}$ substrates (ref. 12 and Brar, V. et al., 2008 APS March Meeting, Abstract: U29.00003, unpublished) will inevitably lead to a broadening of the absorption onset at $2 E_{\mathrm{F}}$ in $\sigma_{1}(\omega)$, because infrared measurements register the absorption averaged over a large area (a few micrometres in our experiments). The origin of the inhomogeneity of $E_{\mathrm{F}}$ in graphene is still an open question $^{12}$, which needs to be explored using spatially resolved probes such as near-field infrared conductivity studies capable of probing the response of a material with nanometre resolution over a large area $^{13}$.

Our study has uncovered several new properties of graphene that are beyond the ideal Dirac fermion picture ${ }^{14}$. First, our study revealed unexpected features of $\sigma_{1}(\omega, V)$ below $2 E_{\mathrm{F}}$. The band structure of ideal graphene implies that the interband transition at $2 E_{\mathrm{F}}$ is the lowest electronic excitation in the system apart from the Drude response at $\omega=0$. Therefore, we anticipate finding $\sigma_{1}(\omega, V) \approx 0$ up to the $2 E_{\mathrm{F}}$ threshold, provided the Drude scattering rate is much smaller than $2 E_{\mathrm{F}}$. This latter condition is fulfilled for all data in Fig. 2, and yet we registered significant conductivity below $2 E_{\mathrm{F}}$ (see the Supplementary Information). This result has not been anticipated by theories developed for Dirac fermions ${ }^{6-8}$. Both extrinsic and intrinsic effects may give rise to the residual conductivity in Fig. 2. Among the former, charged impurities and unitary scatterers (edge defects, cracks, vacancies and so on) were shown to induce considerable residual conductivity below $2 E_{\mathrm{F}}$ (ref. 11). However, the theoretical residual absorption in ref. 11 is systematically suppressed with voltage, whereas this suppression was not observed in our data. In addition, the magnitude of the theoretical residual absorption is smaller compared with experimental values in Fig. 2. Therefore, it is likely that other mechanisms are also responsible for the residual conductivity in our data. One intriguing interpretation of the residual conductivity is in terms of many-body interactions, which are known to produce a strong frequency-dependent quasiparticle scattering rate $1 / \tau(\omega)$. It is predicted theoretically that $1 / \tau(\omega)$ in graphene increases with frequency owing to electron-electron ${ }^{15,16}$ and electron-phonon interactions ${ }^{17}$. The energy-dependent scattering rate initiates a marked enhancement of the conductivity compared with the lorentzian form prescribed by the Drude model. Such an enhancement in mid-infrared frequencies has been observed in many systems ${ }^{18-20}$.

A closer inspection of the voltage dependence of the $2 E_{\mathrm{F}}$ feature uncovers marked departures from the behaviour anticipated within a single particle picture of graphene. To highlight these deviations, we plot $2 E_{\mathrm{F}}$ as a function of the Fermi wave vector $k_{\mathrm{F}}$ based on $k_{\mathrm{F}}=\sqrt{\pi N}=\sqrt{\pi C_{\mathrm{g}} V / e}$, as shown in Fig. 3a. The $E_{\mathrm{F}}\left(k_{\mathrm{F}}\right)$ plot has a clear physical meaning: it is a direct representation of the band dispersion. We then examine the ratio $E_{\mathrm{F}} / \hbar k_{\mathrm{F}}$, which is directly related to the Fermi velocity $v_{\mathrm{F}}$. The $E_{\mathrm{F}} / \hbar k_{\mathrm{F}}$ plot as a function of $V^{1 / 2}$ and $k_{\mathrm{F}}$ in Fig. $3 \mathrm{~b}$ reveals a departure from linear dispersion with a single value of $E_{\mathrm{F}} / \hbar k_{\mathrm{F}}$ expected within a single particle picture. Moreover, $E_{\mathrm{F}} / \hbar k_{\mathrm{F}}$ increases systematically with decreasing $k_{\mathrm{F}}$ values compared with that at high $k_{\mathrm{F}}$ values. The observed systematic enhancement of $E_{\mathrm{F}} / \hbar k_{\mathrm{F}}$ at low $k_{\mathrm{F}}$ is indicative of manybody interactions ${ }^{14,21,22}$. Signatures of band renormalization were also observed in a previous magneto-optical study of graphene ${ }^{4}$. Importantly, even the smallest $E_{\mathrm{F}} / \hbar k_{\mathrm{F}}$ values in Fig. $3 \mathrm{~b}$ are higher than that of the bulk graphite ${ }^{23}\left(\sim 0.9 \times 10^{6} \mathrm{~m} \mathrm{~S}^{-1}\right)$, which also supports the hypothesis of $E_{\mathrm{F}} / \hbar k_{\mathrm{F}}$ renormalization in graphene. Complementary information on the $E_{\mathrm{F}} / \hbar k_{\mathrm{F}}$ renormalization in graphene can be obtained from photoemission, which is another 
potent probe of many-body effects in solids. Currently available photoemission data were all collected for epitaxial graphene grown on $\mathrm{SiC}$ (refs 24,25). This complicates a direct comparison with infrared results for exfoliated samples on $\mathrm{SiO}_{2} / \mathrm{Si}$ substrates reported here. We conclude by noting that the strong deviations of the experimental electromagnetic response from a simple single particle picture of graphene reported in our study challenge current theoretical conceptions of fundamental properties of this interesting form of carbon and also have implications for its potential applications in opto-electronics.

Note added in proof. After the submission of our paper, we became aware of another work on gate tunable infrared properties of graphene $e^{26}$.

\section{METHODS}

\section{SAMPLE FABRICATION AND INFRARED MEASUREMENTS}

In the graphene devices studied here, monolayer graphene mechanically cleaved from Kish graphite was deposited onto an infrared transparent $\mathrm{SiO}_{2}(300 \mathrm{~nm}) / \mathrm{Si}$ substrate ${ }^{2,3}$, which also serves as the gate electrode. Then, standard fabrication procedures were used to define multiple $\mathrm{Cr} / \mathrm{Au}(3 / 35 \mathrm{~nm})$ contacts to the sample. The devices studied here exhibit mobility as high as $8,700 \mathrm{~cm}^{2} \mathrm{~V}^{-1} \mathrm{~s}^{-1}$ measured at carrier densities of $\sim 2 \times 10^{12} \mathrm{~cm}^{-2}$. The characteristic half-integer quantum Hall effect is observed in these samples ${ }^{2,3}$, confirming the single layer nature of our specimen. Infrared experiments were carried out using an infrared microscope operating with a synchrotron source at the Advanced Light Source in the frequency range of $700-8,000 \mathrm{~cm}^{-1}$. The synchrotron beam is focused in a diffraction-limited spot, which is smaller than the sample. We measured the reflectance $R(\omega)$ and transmission $T(\omega)$ of the graphene devices as a function of gate voltage $V_{\mathrm{g}}$ with simultaneous monitoring of the d.c. resistivity.

\section{TEMPERATURE OF THE GRAPHENE SAMPLE}

Data reported here were obtained in a micro-cryostat with the sample mounted on a coldfinger in vacuum. The temperature of our graphene sample is warmer than that of the coldfinger, owing to thermal radiation from room-temperature $\mathrm{KBr}$ optical windows and electrical isolation of the devices from the coldfinger that compromises thermal contact. A sensor mounted in the immediate proximity to the Si substrate of the devices read $T=45 \mathrm{~K}$ at the lowest temperatures attainable at the coldfinger. Because both the temperature sensor and the device are in a nearly identical environment, we assumed this reading to be accurate for graphene as well.

\section{EXTRACTING THE OPTICAL CONSTANTS OF GRAPHENE}

The graphene device contains four layers: (1) graphene with 2D optical conductivity $\sigma(\omega)=\sigma_{1}(\omega)+i \sigma_{2}(\omega)$, (2) $\mathrm{SiO}_{2}$ gate insulator, (3) $\mathrm{Si}$ accumulation layer that forms at the interface of $\mathrm{SiO}_{2} / \mathrm{Si}$ under the applied bias and (4) Si substrate. Properties of layers 2 and 4 are independent of the gate voltage, whereas layers 1 and 3 are systematically modified by $V_{\mathrm{g}}$. In our analysis of these multilayer structures, we followed the protocol detailed in ref. 27. Specifically, we carried out reflection, transmission and ellipsometric measurements on the Si substrates and $\mathrm{SiO}_{2} / \mathrm{Si}$ wafers used in our devices and thus obtained the optical constants of layers 2 and 4 . We then investigated infrared properties of test devices $\mathrm{Ti} / \mathrm{SiO}_{2} / \mathrm{Si}$ as a function of gate voltage and thus extracted the optical constants of the $\mathrm{Si}$ accumulation layer in wafers used for graphene devices. We find that the response of the Si accumulation layer is confined to far-infrared frequencies ${ }^{28}$ and gives negligible contribution to mid-infrared data in Fig. 1. Finally, we used a multi-oscillator fitting procedure ${ }^{27}$ to account for the contribution of $\sigma(\omega)$ of graphene to the reflectance and transmission spectra shown in Fig. 1 using standard methods for multilayered structures.

\section{EXTRACTING FERMI ENERGY EF FROM CONDUCTIVITY SPECTRA}

Because of the broadening of the $2 E_{\mathrm{F}}$ threshold in $\sigma_{1}(\omega, V)$, the $E_{\mathrm{F}}$ values can be determined most accurately from the imaginary part of the optical conductivity spectra $\sigma_{2}(\omega, V)$ shown in Fig. 2c. Indeed, these spectra reveal a sharp minimum at $\omega=2 E_{\mathrm{F}}$ in agreement with a previous theoretical prediction $^{29}$. The minimum in the $\sigma_{2}(\omega, V)$ spectrum is found from the frequency where the derivative of $\sigma_{2}(\omega, V)$ with respect to frequency is zero. The uncertainties of $2 E_{\mathrm{F}}$ obtained from this method are related to the accuracy in defining the minimum in the $\sigma_{2}(\omega, V)$ spectrum. Alternatively, $2 E_{\mathrm{F}}$ values can be extracted from the centre frequency of the $2 E_{\mathrm{F}}$ threshold in $\sigma_{1}(\omega, V)$. The second method has larger uncertainties, as shown in Fig. 3, due to the ambiguity of defining the centre of the $2 E_{\mathrm{F}}$ threshold in $\sigma_{1}(\omega, V)$.

Received 22 January 2008; accepted 6 May 2008; published 8 June 2008.

\section{References}

1. Semenoff, G. W. Condensed-matter simulation of a three-dimensional anomaly. Phys. Rev. Lett. 53, 2449-2452 (1984)

2. Novoselov, K. S. et al. Two-dimensional gas of massless Dirac fermions in graphene. Nature 438, 197-200 (2005)

3. Zhang, Y., Tan, J. W., Stormer, H. L. \& Kim, P. Experimental observation of the quantum Hall effect and Berry's phase in graphene. Nature 438, 201-204 (2005).

4. Jiang, Z. et al. Infrared spectroscopy of Landau levels of graphene. Phys. Rev. Lett. 98, 197403 (2007).

5. Deacon, R. S., Chuang, K.-C., Nicholas, R. J., Novoselov, K. S. \& Geim, A. K. Cyclotron resonance study of the electron and hole velocity in graphene monolayers. Phys. Rev. B 76, 081406 (2007).

6. Ando, T., Zheng, Y. \& Suzuura, H. Dynamical conductivity and zero-mode anomaly in honeycomb lattices. J. Phys. Soc. Jpn. 71, 1318-1324 (2002).

7. Peres, N. M. R., Guinea, F. \& Castro Neto, A. H. Electronic properties of disordered two-dimensional carbon. Phys. Rev. B 73, 125411 (2006).

8. Gusynin, V. P. \& Sharapov, S. G. Transport of Dirac quasiparticles in graphene: Hall and optical conductivities. Phys. Rev. B 73, 245411 (2006).

9. Nair, R. R. et al. Fine structure constant defines visual transparency of graphene. Science doi:10.1126/science.1156965 (2008).

10. Kuzmenko, A. B., van Heumen, E., Carbone, F. \& van der Marel, D. Universal optical conductance of graphite. Phys. Rev. Lett. 100, 117401 (2008).

11. Peres, N. M. R., Stauber, T. \& Castro Neto, A. H. The infrared conductivity of graphene. Preprint at $<$ http://arxiv.org/abs/0803.2816> (2008).

12. Martin, J. et al. Observation of electron-hole puddles in graphene using a scanning single-electron transistor. Nature Phys. 4, 144-148 (2008).

13. Qazilbash, M. M. et al. Mott transition in $\mathrm{VO}_{2}$ revealed by infrared spectroscopy and nano-imaging. Science 318, 1750-1753 (2007).

14. Castro Neto, A. H., Guinea, F., Peres, N. M. R., Novoselov, K. S. \& Geim, A. K. The electronic properties of graphene. Preprint at $<$ http://arxiv.org/abs/0709.1163> (2007).

15. González, J., Guinea, F. \& Vozmediano, M. A. H. Unconventional quasiparticle lifetime in graphite. Phys. Rev. Lett. 77, 3589-3592 (1996).

16. Hwang, E. H., Hu, B. Y.-K. \& Das Sarma, S. Inelastic carrier lifetime in graphene. Phys. Rev. B 76, 115434 (2007).

17. Park, C.-H., Giustino, F., Cohen, M. L. \& Louie, S. G. Velocity renormalization and carrier lifetime in graphene from the electron-phonon interaction. Phys. Rev. Lett. 99, 086804 (2007).

18. Basov, D. N., Singley, E. J. \& Dordevic, S. V. Sum rules and electrodynamics of high- $T_{\mathrm{c}}$ cuprates in the pseudogap state. Phys. Rev. B 65, 054516 (2002).

19. Degiorgi, L. The electrodynamic response of heavy-electron compounds. Rev. Mod. Phys. 71, 687-734 (1999).

20. Basov, D. N. \& Timusk, T. Electrodynamics of high- $T_{\mathrm{c}}$ superconductors. Rev. Mod. Phys. 77, 721-779 (2005).

21. Gonzalez, J., Guinea, F.. \& Vozmediano, M. A. H. Marginal-Fermi-liquid behavior from two-dimensional Coulomb interaction. Phys. Rev. B 59, R2474-R2477 (1999).

22. Das Sarma, S., Hwang, E. H. \& Tse, W.-K. Many-body interaction effects in doped and undoped graphene: Fermi liquid versus non-Fermi liquid. Phys. Rev. B 75, 121406 (2007).

23. Zhou, S. Y. et al. First direct observation of Dirac fermions in graphite. Nature Phys. 2 , 595-599 (2006).

24. Bostwick, A., Ohta, T., Seyller, T., Horn, K. \& Rotenberg, E. Quasiparticle dynamics in graphene. Nature Phys. 3, 36-40 (2006).

25. Zhou, S. Y. et al. Substrate-induced bandgap opening in epitaxial graphene. Nature Mater. 6 770-775 (2007).

26. Wang, F. et al. Gate-variable optical transitions in graphene. Science 320, 206-209 (2008).

27. Li, Z. Q. et al. Light quasiparticles dominate electronic transport in molecular crystal field-effect transistors. Phys. Rev. Lett. 99, 016403 (2007).

28. Sai, N., Li, Z. Q., Martin, M. C., Basov, D. N. \& Di Ventra, M. Electronic excitations and metal-insulator transition in poly(3-hexylthiophene) organic field-effect transistors. Phys. Rev. B 75, 045307 (2007)

29. Mikhailov, S. A. \& Ziegler, K. New electromagnetic mode in graphene. Phys. Rev. Lett. 99 , 016803 (2007).

Supplementary Information accompanies this paper on www.nature.com/naturephysics.

\section{Acknowledgements}

Work at UCSD is supported by the DOE (No. DE-FG02-00ER45799). Research at Columbia University is supported by the DOE (No. DE-AIO2-04ER46133 and No. DE-FG02-05ER46215), NSF (No. DMR-03-52738 and No. CHE-0117752), NYSTAR and the Keck Foundation. The Advanced Light Source is supported by the Director, Office of Science, Office of Basic Energy Sciences, of the US Department of Energy under Contract No. DE-AC02-05CH11231.

\section{Author information}

Reprints and permission information is available online at http://npg.nature.com/reprintsandpermissions. Correspondence and requests for materials should be addressed to Z.Q.L. 